\title{
O papel da língua na resolução de enunciados matemáticos
}

\author{
Jessica BARCELLOS ${ }^{1}$ \\ Erica dos Santos RODRIGUES ${ }^{2}$ \\ Cilene RODRIGUES 3
}

\footnotetext{
${ }^{1}$ Pontifícia Universidade Católica (PUC Rio), jessybarcellos@gmail.com, orcid.org/oooo0001-9084-4802.

${ }^{2}$ Pontifícia Universidade Católica (PUC Rio), ericasr@puc-rio.br, orcid.org/o0oo-00023524-5820.

3 Pontifícia Universidade Católica (PUC Rio), cilene@gmail.com, orcid.org/000০-০oo15324-7486.
} 
RESUMO Este trabalho explora a interface linguagem-matemática, com foco em tarefas de resolução de problemas de divisão partitiva e por quotas. Investiga-se em que medida dificuldades nesses tipos de divisão podem estar relacionadas à complexidade linguística dos enunciados. Foram conduzidos dois experimentos com alunos do $2^{\mathrm{o}}$ ano do Ensino Fundamental de uma escola da rede pública federal de ensino no Rio de Janeiro. No primeiro experimento, foram utilizados como itens experimentais enunciados tal como apresentados em livros didáticos utilizados no ensino público, e os resultados indicam diferença significativa entre divisão partitiva e divisão por quotas, com maior número de acertos em divisão partitiva. No segundo experimento, enunciados novos foram elaborados, com controle da estrutura informacional e da complexidade gramatical nos dois tipos de problemas. Os resultados indicam desempenho similar tanto em divisão partitiva quanto por quotas. A análise conjunta dos dois experimentos indica que a dificuldade dos alunos na resolução de problemas de divisão pode ser reduzida com controle da complexidade gramatical do enunciado, o que mostra o papel fundamental da observação de variáveis linguísticas na aferição de conhecimento matemático e na elaboração de materiais didáticos. 
ABSTRACT This paper investigates the crossdomain language-mathematics, focusing on problems of division involving partitive and quotative conditions. We investigate whether the difficulties students face when solving division tasks can be related to the linguistic complexity of the verbal commands. Two experiments were conducted with secondgrade students of a primary school in Rio de Janeiro. In the first experiment, commands for division problems extracted from mathematics textbooks were used as experimental items. The results indicate a significant difference between partitive and quotative conditions, as participants were significantly better on partitive division. In the second experiment, new commands were elaborated, controlling for the informational structure as well as for grammatical complexity. The results show a similar performance in both conditions. Overall, the obtained results indicate that difficulties students usually face in solving mathematical problems might be related to how commands are formulated linguistically, especially to issues related to grammatical complexity. This points towards the necessary of controlling linguistic variables when mathematical and logical reasoning are being evaluated. 
PALAVRAS-CHAVE Resolução de problemas de matemática. complexidade gramatical. interface linguagem-matemática.

KEYWORDS problem solving. grammatical complexity. interface language-mathematics. 


\section{Introdução}

A investigação da interface linguagem-matemática é um campo de estudos que pode contribuir diretamente para as práticas pedagógicas na Educação Básica. No entanto, apesar dessa relevância e aplicabilidade, há poucos estudos que, do ponto de vista linguístico e psicolinguístico, investigam o papel das estruturas linguísticas no processo de resolução de atividades matemáticas.

Neste trabalho, reportamos resultados parciais da pesquisa realizada por Barcellos (2017), na qual se buscou explorar em que medida as estruturas linguísticas utilizadas nos enunciados podem influenciar na interpretação de enunciados de problemas matemáticos realizada pela criança, e consequentemente as estratégias de resolução.

Elaborada dentro da linha pesquisa sobre linguagem e cognição, nossa hipótese de trabalho é que a complexidade gramatical das estruturas usadas nos enunciados afeta a interpretação da situaçãoproblema, o que se reflete no desempenho na tarefa. Na pesquisa realizada, foram examinadas situações-problemas envolvendo divisão partitiva e divisão por quotas. Estes são os tipos de divisão mais enfatizados na educação básica, sendo a segunda considerada, por alguns autores, mais complexa em termos do tipo de raciocínio matemático a ser implementado. Nos livros didáticos, contudo, não há preocupação com onível de complexidade sintática dos enunciados dos problemas, e, em geral, as estruturas usadas nos problemas de divisão por quotas são mais complexas em termos sintáticomorfológicos e/ou sintático-semânticos do que aquelas empregadas nos problemas de divisão partitiva. Buscamos, então, verificar em que medida esse fator pode contribuir para uma possível diferença de desempenho na resolução dos dois tipos de problemas. Nossa previsão é que, ao se neutralizarem diferenças linguísticas entre os enunciados a partir do emprego de estruturas com menor nível de 
complexidade gramatical, neutralizam-se também as diferenças de desempenho na resolução dos tipos de divisão em consideração.

O trabalho busca um diálogo entre os construtos teóricos da Linguística Gerativista e da Psicolinguística e estabelece interface com as áreas da Cognição matemática, da Educação matemática e da Psicologia Cognitiva.

O presente artigo se organiza da seguinte maneira: a seção 1 é dedicada a questões inerentes à interface linguagem-matemática; a seção 2 contém uma caracterização dos tipos de enunciados de divisão considerados na pesquisa; a seção 3 traz discussões sobre a interferência da linguagem na resolução de problemas e os resultados dos dois experimentos realizados; e a seção 4 contém as considerações finais.

\section{Interface entre linguagem e matemática}

A resolução de problemas matemáticos, por implicar na interligação de componentes lógico-matemáticos e componentes linguísticos, se constitui como um objeto relevante para a investigação da interface linguagem-matemática. No âmbito das pesquisas na área de Educação, Lorensatti (2009) e Cândido (2011) reforçam a ideia de que o enunciado do problema deve ser abordado também linguisticamente, pois é possível atribuir à língua dois papéis em relação à matemática. A língua é tanto o código no qual são lidos os enunciados, na qual são feitos os comentários e a qual permite interpretar o que se ouve ou lê de modo preciso ou aproximado, como é também parcialmente aplicada no trabalho matemático, já que os elos de raciocínio matemático apoiam-se na língua, em sua organização sintática e em seu poder dedutivo. Ainda na área da educação matemática, Fruet (2003) ressalta que, algumas vezes, o aluno não resolve adequadamente problemas de matemática não por não saber matemática, e sim por não saber ler ou por não conseguir 
compreender o enunciado do problema. Ocorre que a criança pode saber resolver as operações matemáticas básicas, porém, ao ler o problema, não sabe ou não entende o que deve ser feito. Concluise, dessa discussão, que os processos de resolução de problemas matemáticos ancoram-se no processamento da linguagem.

A interface linguagem-matemática não é um objeto de investigação exclusivo da área de educação matemática. Há também importantes contribuições no âmbito da Psicologia Cognitiva. Destacamos aqui o trabalho de Krutetskii (1976, apud BRITO, FINI; GARCIA, 1994). O autor ressalta que a compreensão do enunciado não é suficiente para resolver problemas matemáticos. Segundo o autor, um nível elevado de desenvolvimento do pensamento lógicoverbal, embora seja uma condição necessária para a resolução do problema, não determina, por completo, a capacidade matemática, sendo necessárias também outras habilidades, como por exemplo: flexibilidade do pensamento matemático, habilidade para alcançar e abreviar passos da resolução e memória específica para elementos matemáticos. Por outro lado, o autor argumenta também que baixo nível no raciocínio lógico-verbal tende a dificultar a compreensão e a habilidade matemática, uma vez que a compreensão do enunciado é anterior à compreensão da natureza matemática do problema.

O também psicólogo cognitivo Richard Mayer, em seu texto clássico Thinking, problem solving and cognition (1992), aponta que a compreensão do enunciado é o primeiro passo para sua resolução de um problema matemático. Segundo Mayer, para compreender uma questão matemática, o sujeito precisa traduzir a linguagem expressa em informações matemáticas, o que requer o uso de 3 tipos de conhecimento: linguístico, semântico e esquemático. $\mathrm{O}$ conhecimento linguístico refere-se ao conhecimento da estrutura gramatical da língua na qual o enunciado é apresentado, à compreensão e interpretação desta e ao estabelecimento de relações 
entre linguagem e as informações matemáticas. $\mathrm{O}$ conhecimento semântico é caracterizado pela maneira como a linguagem codifica e expressa fatos e entidade do mundo, possibilitando também a realização de inferências semânticas e pragmáticas, através do acionamento de conhecimentos aprendidos no dia a dia. $\mathrm{O}$ conhecimento esquemático informa o leitor sobre qual é o tipo de problema a ser resolvido, isto é, quais são os dados úteis e quais são as ações necessárias para obter a resolução. Os esquemas se constituem como conhecimentos representados na memória e são essenciais para a resolução de novas situações-problemas.

Pesquisas com foco em dificuldades de aprendizagem, têm buscado adaptar a linguagem utilizada nos enunciados matemáticos (ABEDI, LORD \& HOFSTETTER, 1998; ABEDI \& LORD, 2001; MARTINELLO, 2009). Os poucos trabalhos que se ocupam desse tema fazem adaptações linguísticas, prioritariamente, buscando reduzir a complexidade lexical (e.g. número de palavras de baixa frequência, palavras ambíguas e polissêmicas, expressões idiomáticas) e a complexidade sintática (e.g. tamanho das sentenças, tamanho dos sintagmas nominais, número de sintagmas preposicionados, número de modificadores no particípio e presença de sentenças sintaticamente complexas, como relativas, completivas, adverbiais e condicionais).

Abedi\&Lord (2001), porexemplo,conduziramdoisexperimentos nos quais as estruturas linguísticas presentes em enunciados do Programa Nacional de Avaliação em Matemática dos EUA (National Assessment of Educational Progress - NAEP) foram manipuladas, com o objetivo de reduzir a complexidade lexical e sintática das sentenças. As modificações realizadas consistiram, basicamente, no uso de vocabulário mais frequente, na mudança da voz verbal passiva para ativa, na redução de sintagmas nominais, na substituição de orações condicionais e relativas por orações coordenadas. 
No primeiro experimento, um total de 36 alunos da $8^{\mathrm{a}}$ série do Ensino Fundamental de quatro escolas de Los Angeles, foram instruídos a selecionar, dentre duas situações-problema, qual escolheriam para responder em uma prova com limite de tempo. Os alunos preferiram os enunciados modificados (63\%) aos enunciados originais (37\%). No segundo experimento, 1174 alunos da $8^{a}$ série do Ensino Fundamental de 11 escolas de Los Angeles foram instruídos a resolver 25 problemas de matemática, no período de uma hora. Dos 25 estímulos, 10 eram enunciados originais, 10 eram modificados e 5 distratores. O nível socioeconômico dos alunos (maior e menor poder aquisitivo), o nível de proficiência na língua inglesa (falantes nativos de inglês e falantes de inglês como $2^{\mathrm{a}}$ língua) e o nível de desempenho nas aulas de matemática (desempenho regular e alto desempenho) foram definidas como variáveis grupais. Verificouse que a média de acerto dos participantes, no geral, foi de 14,01. A diferença entre o número de acertos nos itens modificados e nos itens originais foi estatisticamente significativa $(\mathrm{p}=.003)$, com desempenho mais elevado nos itens modificados. O grupo de estudantes falantes nativos de inglês apresentou maior taxa de acerto $(15,14)$ quando comparado ao grupo de alunos que tinha inglês como segunda língua $(11,56)$. A comparação entre as variáveis classe econômica indicou que alunos de classes sociais mais favorecidas também tiveram maior taxa de acerto $(14,96)$ do que os alunos de níveis desfavorecidos socioeconomicamente $(12,47)$, e o nível de desempenho nas aulas de matemática foi a variável que gerou diferenças mais expressivas, tendo a taxa de acerto variado de 5,21 nas turmas de menor desempenho a 21,33 nas turmas mais avançadas.

Em resumo, os resultados dos experimentos apresentados acima indicam que a estrutura linguística dos enunciados afeta $o$ desempenho dos alunos. Os autores pontuam que tais resultados ilustram a relação existente entre habilidades de leitura e resolução 
de problemas matemáticos e argumentam que é fundamental que a relação entre linguagem e cognição matemática seja um ponto crucial na pesquisa e práticas no campo da educação matemática.

No âmbito da Psicolinguística, o número de trabalhos que exploram a interface linguagem-matemática é reduzido. Correia (2004 e 2013) é uma das poucas autoras a realizar trabalhos nessa linha de investigação em Língua Portuguesa. Partindo do pressuposto teórico de que construções que apresentam maior grau de complexidade sintática induzem um baixo nível de compreensão, a autora investigou de que forma estruturas passivas afetam o processo de compreensão dos enunciados de problemas de matemática. $\mathrm{O}$ estudo foi aplicado a alunos portugueses de diferentes níveis de escolaridade $\left(4^{\circ}, 6^{\circ}\right.$ e $9^{\circ}$ anos). Para compor o corpus do experimento, foram selecionados exercícios de manuais didáticos utilizados em diferentes anos de escolaridade do ensino fundamental. Os enunciados foram organizados em três testes, sendo cada teste formado por quatro exercícios em que predominavam, respectivamente, estruturas ativas, estruturas passivas perifrásticas e passivas pronominais. Além de resolverem o enunciado matemático, os participantes foram submetidos a uma tarefa linguística, que consistia em responder a perguntas de compreensão sobre o enunciado, como "quem ofereceu?"; "o que foi oferecido?” e “a quem se ofereceu?”. A adição dessa tarefa teve como objetivo verificar se os participantes interpretavam corretamente os enunciados, identificando os argumentos nucleares e opcionais do verbo.

Todos os grupos obtiveram melhor desempenho no teste com estruturas ativas do que com passivas, sendo que os resultados no teste envolvendo passivas pronominais foram piores do que os do teste com passivas perifrásticas. A diferença de desempenho entre esses tipos de estrutura só foi significativa para o grupo de $2^{\circ}$ ano, 
mas a mesma tendência foi observada entre alunos dos demais anos escolares. Verificou-se ainda que todos os alunos gastaram mais tempo na resolução de problemas com passivas pronominais. $\mathrm{Na}$ tarefa de compreensão linguística, foram encontrados resultados similares, havendo melhor desempenho, em todos os grupos, em estruturas com ativas e índice mais baixo de acertos com estruturas passivas pronominais. A autora sugere que um possível motivo para o baixo desempenho nos com passivas (pronominais e perifrásticas) foi a ausência de um argumento com função semântica de agente.

A relação entre o processo de compreensão de estruturas passivas e o nível de escolarização dos participantes também foi avaliada. Os resultados mais baixos, tanto no domínio da compreensão quanto no de raciocínio lógico, foram obtidos pelos alunos do $4^{\circ}$ ano, com melhores resultados em estruturas ativas, seguido de estruturas perifrásticas e, por último, estruturas pronominais. Nos demais grupos, a mesma tendência foi observada, mas com redução no número de erros. Portanto, conforme defende a autora, esses resultados também demonstram que o desempenho em tarefas de resolução de problemas matemáticos é condicionado pelo tipo de estrutura sintática na qual o enunciado é elaborado. No entanto, é preciso ressaltar que a falta de um controle mais robusto nos estímulos utilizados é um ponto crítico do trabalho de Correia. Nenhuma operação aritmética específica foi considerada e não foram controladas as razões numéricas utilizadas nos problema. Além disso, é preciso apontar que a complexidade dos conteúdos trabalhados em cada um dos anos de escolaridade testados é bastante diversa, o que pode ter interferido nos resultados obtidos. De qualquer modo, os resultados de Correia sugerem que a interferência da complexidade gramatical dos enunciados diminui com a experiência escolar, de modo que os alunos das séries mais avançadas não são tão beneficiados por um possível controle de variáveis linguísticas quanto os alunos mais novos. 
Apesar de não analisarmos o mesmo tipo de estrutura linguística que Correia (cf. seção 3), destacamos o trabalho da autora como sendo um dos poucos que busca manipular complexidade gramatical para avaliar o efeito da língua no desempenho de tarefas de resolução de problemas matemáticos.

No presente trabalho, apresentamos dois experimentos em que se buscou verificar efeito de uniformização das estruturas linguísticas utilizadas nos problemas de divisão apresentados para alunos do $2^{\circ}$ ano do Ensino Fundamental. Nesse ano de escolaridade, além de ainda estarem se familiarizando com a tarefa de resolução de problemas escritos, os alunos são iniciados no ensino formal da operação da divisão. Dessa forma, levando em consideração os experimentos discutidos acima, em especial o de Correia, que sugere uma interação entre grau de escolaridade e dificuldade linguística, acreditamos que a adaptação linguística dos enunciados pode ser indicativa da natureza das dificuldades apresentadas por alunos do segundo ano: se decorrentes de questões lógico-matemáticas ou se decorrentes de dificuldades de leitura e compreensão.

\section{Tipos de enunciados de divisão}

A divisão, uma das quatro operações fundamentais da matemática, ocupa um lugar de destaque no currículo do Ensino Fundamental 1, nas escolas brasileiras. Há diferentes conceitos de divisão a serem trabalhados. Os mais enfatizados no $1^{\circ}$ ciclo do ensino fundamental são a divisão partitiva e a divisão por quotas.

A divisão como distribuição também pode ser nominada de divisão como partilha e/ou divisão partitiva. Carpenter et al. (1999) definiu esse tipo de divisão como uma situação em que uma quantidade é partilhada igualmente entre um dado número de receptores e pedimos para determinar quantos objetos há para cada receptor. Nesse tipo de problema, o todo (total de elementos) 
é conhecido, assim como o número de partes em que o todo será dividido, e o resultado deve ser determinado pelo tamanho de cada parte. Há sempre duas grandezas diferentes dadas no enunciado e que precisam ser relacionadas para a obtenção do resultado. Exemplo de enunciado envolvendo esse conceito de divisão:

1. Maria decidiu distribuir 30 figurinhas (TOTAL DE ELEMENTOS) entre 3 crianças (PARTES). Quantas figurinhas cada uma receberá? (TAMANHO DAS PARTES)

Grandezas envolvidas no problema

30 FIGURINHAS (todo)

3 CRIANÇAS (número de partes)

Grandezas envolvidas no resultado:

10 FIGURINHAS (tamanho das partes)

Já na divisão como medida, também denominada de divisão por quotas ou quotativa, busca-se verificar quantas vezes uma determinada quantidade "cabe" em outra. Esse tipo de divisão referese a uma situação-problema em que o total de elementos e o tamanho de cada parte são conhecidos e é preciso determinar a quantidade de partes em que o todo foi dividido. Nesse tipo de problema, trabalhase apenas com uma grandeza nos dados numéricos do enunciado. Por exemplo: 
2. Maria decidiu distribuir 30 figurinhas (TOTAL DE ELEMENTOS) entre as crianças de sua rua (PARTES). Ela deu 10 figurinhas para cada criança. (TAMANHO DAS PARTES). Quantas crianças receberam figurinhas? (QUANTAS PARTES) Grandezas envolvidas no problema: 30 FIGURINHAS (todo) 10 FIGURINHAS (tamanho das partes) Grandezas envolvidas no resultado 3 CRIANÇAS (número de partes)

$\mathrm{Na}$ área de cognição matemática, não há consenso a respeito de qual dos dois tipos de problema exigiria um raciocínio matemático mais complexo. Como veremos a seguir, as pesquisas apontam em mais de uma direção, tendo assim diferentes consequências para o ensino formal da divisão.

Pesquisadores como Hill (1952), Brown (1981a), Burton (1992) e Downtown (2009) consideram que há pouca diferença entre o grau de dificuldade dos dois tipos de problemas, uma vez que a estrutura da operação é bem similar nos dois modelos, sendo um o complemento do outro.

Downtown (2009) também defende a mesma posição a partir de dados experimentais. A autora conduziu um estudo com 26 alunos do $3^{\circ}$ ano de escolas de públicas de Melbourne/Austrália. O objetivo do experimento foi verificar como estudantes do $3^{\circ}$ ano resolvem problemas de divisão partitiva e divisão por quotas, observando se há diferença na resolução. Os resultados indicaram equivalência nos dois tipos de divisão tanto na acurácia das respostas como nas estratégias de resolução. 
No entanto, há trabalhos que apontam diferenças na complexidade cognitiva nesses dois conceitos, sendo que a divisão por quotas é tomada como mais fácil do que a divisão partitiva (GUNDERSON, 1955; ZWENG, 1964; BURGEOIS \& NELSON, 1977; MAMEDE \& VASCONCELLOS, 2016). Gunderson (1955) e Zweng (1964), por exemplo, apontam que o ensino formal deve partir dos problemas quotativos, uma vez que calcular o número de partes em que o todo foi dividido envolve raciocínio menos complexo do que o exigido nos problemas de divisão partitiva, por permitir estratégia de subtrações sucessivas para verificar quantas vezes uma quantidade $X$ está contida em uma quantidade Y. Essas conclusões baseiam-se em estudos experimentais com $2^{\circ}$ Ano do Ensino Fundamental, em que se verificou maior taxa de acertos em divisão por quotas do que nos de divisão partitiva.

Mamede e Vasconcellos (2016) também encontraram resultados nessa direção. As autoras conduziram experimentos com 42 alunos do $4^{\circ}$ ano (entre 9 e 10 anos) de uma escola pública em Braga, Portugal, com o objetivo de investigar a compreensão da relação inversa entre divisor e quociente nos problemas de divisão partitiva e por quotas. Os resultados obtidos indicam que houve maior número de acertos nos problemas de divisão por quotas do que nos de divisão partitiva. Segundo as autoras, os resultados indicam que a relação entre os termos da divisão é expressa de maneira mais clara em enunciados de divisão por quotas do que em enunciados partitivos, o que facilita o desempenho dos alunos.

Por outro lado, pesquisadores clássicos na área da Educação Matemática,como Fischbein,Deri,Nello\&Marino (1985), sustentam, por meio de evidências empíricas e argumentos epistemológicos que a divisão partitiva é mais facilmente compreendida pelas crianças do que a divisão por quotas. Considera-se que o modelo intuitivo e primitivo de divisão, que corresponde a características primárias, 
naturais e básicas do ser humano, é a divisão partitiva, sendo a divisão por quotas adquirida posteriormente, por meio da instrução de caráter formal. Como consequência, sugere-se que o ensino da divisão deva ser iniciado via divisão partitiva para que a complexidade cognitiva associada à resolução de problemas matemáticos cresça gradativamente com o auxílio do ensino formal. Nessa linha de argumentação, Brown (1981b) verificou que, em tarefas nas quais se solicita a criação de um problema que corresponda a uma sentença matemática de divisão, as crianças tendem a elaborar problemas partitivos e não problemas de divisão por quotas. Segundo o autor, isso se deve ao fato de as crianças associarem, desde muito pequenas, o sinal de divisão à ideia de repartir uma quantidade $\mathrm{X}$ em um número Y de partes (associada a problemas de divisão partitiva) e não à ideia de medir quantas vezes um Y cabe em um X (associada problemas de divisão por quotas).

Fischbein, Deri, Nello \& Marino (1985) conduziram um estudo com alunos de $5^{\circ}, 7^{\circ}$ e $9^{\circ}$ anos de 13 escolas de Pisa, Itália. Os alunos foram instruídos a resolver um teste com 42 problemas (12 de multiplicação, 14 de divisão e 16 distratores (adição e subtração). Foi pedido aos participantes que apenas indicassem qual operação solucionaria o problema, não sendo necessária a resolução real do problema. Nos enunciados de divisão, foram utilizados problemas de divisão partitiva e de divisão quotativa. Os resultados obtidos indicaram que os problemas de divisão partitiva foram facilmente solucionados pelos alunos do $7^{\circ}$ e do $9^{\circ}$ ano. Apenas os alunos mais novos, do $5^{\circ}$ ano, tiveram dificuldades com esse tipo de enunciado. Os autores apontam que tais dificuldades podem ter sido acarretadas pela presença de numerosidades maiores (e.g 1500) e pelo uso de abreviações possivelmente não conhecidas pelos alunos, como unidades de massa e de comprimento (e.g kg e km). Já os problemas de divisão por quotas só foram solucionados corretamente pelogrupo 
de alunos mais velhos $-9^{\circ}$ ano. Segundo os autores, tais resultados evidenciam que, apenas após a instrução formal, os alunos adquirem o modelo de divisão por quotas, podendo, então resolver esse tipo de problema com menos dificuldade.

Os autores apontam ainda que a escolha didático-metodológica de utilizar a adição repetitiva como modelo para o ensino da multiplicação e de iniciar o ensino da divisão a partir dos problemas de partição e só posteriormente apresentar divisão por quotas é uma estratégiafundamentada em razões epistemológicas eontogenéticas, uma vez que esses modelos correspondem melhor aos requisitos mentais dos alunos nos anos iniciais de escolarização. Ou seja, se a divisão partitiva é o modelo prototípico da operação de divisão, ela é mais facilmente compreendida até mesmo por crianças que ainda não foram expostos ao ensino formal da divisão, facilitando a compreensão do conceito de quotas e dos demais modelos associados à divisão.

Em resumo, parece não haver consenso na área de Educação Matemática sobre qual tipo de divisão envolve maior esforço cognitivo. No entanto, um aspecto a ser considerado é tipo de estrutura linguística utilizada na elaboração de problemas partitivos e por quotas. As pesquisas convergem em apontar, uma correlação entre a estrutura linguística utilizada na elaboração do enunciado e o desempenho dos alunos na resolução do problema dado.

\section{Interferência da linguagem na resolução de problemas}

Conduzimos dois experimentos com alunos $2^{\circ}$ ano do ensino fundamental, com o objetivo de testar em que medida a complexidade gramatical dos enunciados afeta a resolução de problemas matemáticos de divisão partitiva e por quotas. Esses experimentos foram diferentes, mas complementares. No experimento 1, 
avaliamos como os alunos resolvem problemas de divisão com enunciados não controlados linguisticamente (enunciados extraídos de livros didáticos). No experimento 2, avaliamos como os alunos resolvem problemas de divisão com enunciados seguindo um padrão linguístico, com controle da complexidade gramatical ${ }^{1}$.

As principais questões linguísticas tratadas nos dois experimentos foram: tipo de sintagma nominal na posição de sujeito da oração que descreve o processo de divisão (relação divisorquociente), presença de estruturas com subordinação sintática, presença de fatores semânticos e pragmáticos que podem alterar o raciocínio lógico, como, por exemplo, referência nominal indefinida, estruturas sintáticas complexas, e tempo irrealis.

Tome, como primeiro exemplo, o problema em (1) retirado de um dos livros didáticos utilizados no experimento 1. A presença da expressão nominal com coordenação de dois nomes próprios força uma leitura semântica ambígua, em que tanto é possível considerar que Sergio e Sandra ganharam 8 balas juntos, ou que ganharam separadamente, somando, portanto, um total de 16 balas. Essa ambiguidade pode dificultar a resolução do problema. Observe ainda a presença da oração relativa dentro do sintagma nominal alvo da divisão [8 balas [ ${ }_{\mathrm{RC}}$ que ganharam $]$ ]. Como se sabe, estruturas sintáticas de relativização envolvem maior custo de processamento (GIBSON, 2000; WARREN \& GIBSON, 2002), podendo, portanto, interferir no processo de resolução da divisão em questão.

(1) Sérgio e Sandra dividiram as 8 balas que ganharam em dois grupos iguais. Com quantas balas cada criança ficou?

\footnotetext{
${ }^{1}$ Para que os alunos participassem dos experimentos, a escola forneceu um parecer favorável à realização da pesquisa e os responsáveis dos alunos assinaram um termo de consentimento livre e esclarecido, autorizando que seus filhos participassem das atividades deste estudo. Além disso, os próprios alunos assinaram um termo de assentimento, no qual foram informados, em linguagem compatível à faixa etária, sobre os objetivos da pesquisa, as tarefas que precisariam realizar e confirmavam se gostariam ou não de participar dos testes.
} 
No exemplo (2) abaixo, também retirado de um dos livros didáticos utilizados no experimento 1 , observam-se várias questões envolvendo os componentes sintaxe, semântica e pragmática. Primeiramente, o uso do sintagma nominal definido as crianças (para distribuir às crianças), sem nenhum antecedente prévio, pode dificultar o processo de entendimento do todo, já que não há como resgatar a entidade a qual a expressão as crianças se refere. $\mathrm{O}$ uso de oração condicional também pode trazer dificuldade de intepretação, já que essas estruturas implicam em possíveis questões de processamento, que podem interferir na resolução do problema apresentado. Primeiramente, a criança precisa considerar a relação semântico-estrutural entre o evento antecedente (nós damos 9 tampinhas para cada criança) e o evento consequente (número de crianças que recebem tampinhas). Na computação dessa relação, o aprendiz de divisão precisará também considerar que, apesar de se tratar de tempo irrealis (engatilhado pela oração condicional), a divisão deverá ser solucionada como se a situação fosse realis. Ou seja, em casos de estruturas envolvendo condicionais, é preciso dar um passo semântico de abstração e considerar os dois eventos relacionados como sendo reais. Se isso não acontecer, corre-se o risco de responder ao problema da seguinte maneira: "Não sei, porque não sei se as crianças receberam tampinhas.”

(2) Temos 27 tampinhas de refrigerantes para distribuir às crianças. Quantas delas receberão as tampinhas se dermos 9 unidades para cada uma?

Portanto, comoveremos, no experimento 2 buscamos controlar a complexidade linguística dos enunciados, reduzindo a complexidade especialmente nos componentes da gramática responsáveis pela interpretação:sintaxe,semânticaepragmática.Oobjetivofoiverificar a possível interferência deste tipo de complexidade na resolução 
de problemas de divisão. Portanto, todos os itens experimentais evitaram sintagmas nominais complexos (e.g. coordenados) e sem referência clara, estruturas de relativização e estruturas envolvendo tempo irrealis. (3) exemplifica um dos itens experimentais utilizado no experimento 2:

(3) Henrique coleciona carta Pokémon. Ele possui 12 cartas e precisa arrumá-las em envelopes. Henrique não sabe qual é o número de envelopes ele precisa para colocar 4 cartas em cada envelope. Você pode ajudá-lo?

No que se segue, apresentaremos os dois experimentos realizados.

\subsection{Experimento 1}

Quando problemas partitivos e quotativos são elaborados sem um controle da complexidade gramatical, há diferença no desempenho dos alunos na resolução desses dois tipos de enunciados? Para responder essa pergunta, realizamos um experimento com uso de situações-problema retirados de livros didáticos adotas em escolas públicas brasileiras. Buscamos com esse experimento ter um parâmetro para comparação com situações de enunciados com estrutura gramatical controlada. A variável independente manipulada foi o tipo de divisão - 4 itens para divisão partitiva e 4 para divisão por quotas. A variável dependente medida foi a taxa de acerto.

\section{Participantes}

Participaram do estudo 20 alunos do $2^{\circ}$ ano do Ensino Fundamental (12 meninas e 8 meninos) deuma escola pública do Rio de Janeiro, com média de idade de 7,6 anos, que ainda não tinham sido apresentados ao ensino sistematizado da operação matemática da divisão. Também 
participaram do experimento, como grupo controle, 12 adultos (7 mulheres e 5 homens), com média de idade de 29,6 anos.

\section{Estímulos}

Foram utilizados 18 enunciados matemáticos, sendo dois de treino, 8 distratores e 8 itens experimentais. Os itens distratores eram compostos por enunciados de adição, subtração e multiplicação. Todos os estímulos foram retirados aleatoriamente de três livros didáticos ${ }^{2}$ participantes do Programa Nacional do Livro didático (PNLD 2016). Para reduzir o número de itens experimentais e, consequentemente, reduzir efeitos de cansaço e fadiga, o teste foi dividido em duas etapas, contendo 9 enunciados cada, e foram realizadas em dias diferentes. Todos os alunos resolveram os mesmos problemas apresentados em ordem aleatória. A tabela abaixo apresenta exemplos dos estímulos utilizados.

Quadro 1: exemplos de estímulos usados no experimento 1

\begin{tabular}{|l|l|}
\hline Tipo de divisão & Enunciado \\
\hline \multirow{5}{*}{ Partitiva } & $\begin{array}{l}\text { Sérgio e Sandra dividiram as 8 balas que ganharam em dois } \\
\text { grupos iguais. Com quantas balas cada criança ficou? }\end{array}$ \\
\cline { 2 - 3 } Quotativa & $\begin{array}{l}\text { Roberto montou 2 porta-lápis para guardar seus 12 lápis de } \\
\text { cor. Quantos lápis haverá em cada porta-lápis se Roberto } \\
\text { colocar a mesma quantidade em cada um deles? }\end{array}$ \\
\hline & $\begin{array}{l}\text { Vou colar 20 adesivos no meu caderno, sendo 4 adesivos em } \\
\text { cada página. Quantas páginas do caderno terão adesivos? }\end{array}$ \\
\cline { 2 - 2 } & $\begin{array}{l}\text { Temos 27 tampinhas de refrigerante para distribuir às } \\
\text { crianças. Quantas delas receberão as tampinhas se dermos 9 } \\
\text { unidades para cada uma? }\end{array}$ \\
\hline
\end{tabular}

\footnotetext{
${ }^{2}$ BORDEAUX, A.; RUBINSTEIN, C.; FRANÇA, E.; et al. Bem-me-quer Alfabetização matemática. São Paulo: Editora BRASIL, 2011.

GARCIA, J. Aprender, muito prazer. Curitiba: Base editorial, 2014.

LONGEN, A. Projeto Jimboê - Alfabetização Matemática. São Paulo: Editora Brasil, 2014.
} 


\section{Procedimento}

Os alunos foram instruídos a resolver os enunciados por meio de desenhos. O registro por meio de desenho é uma técnica muito utilizada nos estudos acerca da cognição matemática, pois ilustra a compreensão das crianças sobre um determinado enunciado. Segundo Smole \& Diniz (2001), o desenho é um pensamento visual que pode adaptar-se a qualquer natureza do conhecimento, e emerge como uma linguagem para a criança, assim como o são o gesto ou a fala. Os alunos realizaram a atividade de forma autônoma, sem mediação do professor. Não foi estabelecido limite de tempo e os alunos levaram, em média, uma hora para resolver cada parte o teste. O grupo controle realizou as duas partes do teste no mesmo momento e levou cerca de 30 minutos para concluir a tarefa.

\section{Resultados}

A taxa total de acertos na condição de divisão partitiva foi maior do que na condição de divisão por quotas $(78,75 \%$ e $61,25 \%$, respectivamente) como é possível verificar no gráfico abaixo.

Gráfico 1: Taxa de acertos no experimento 1

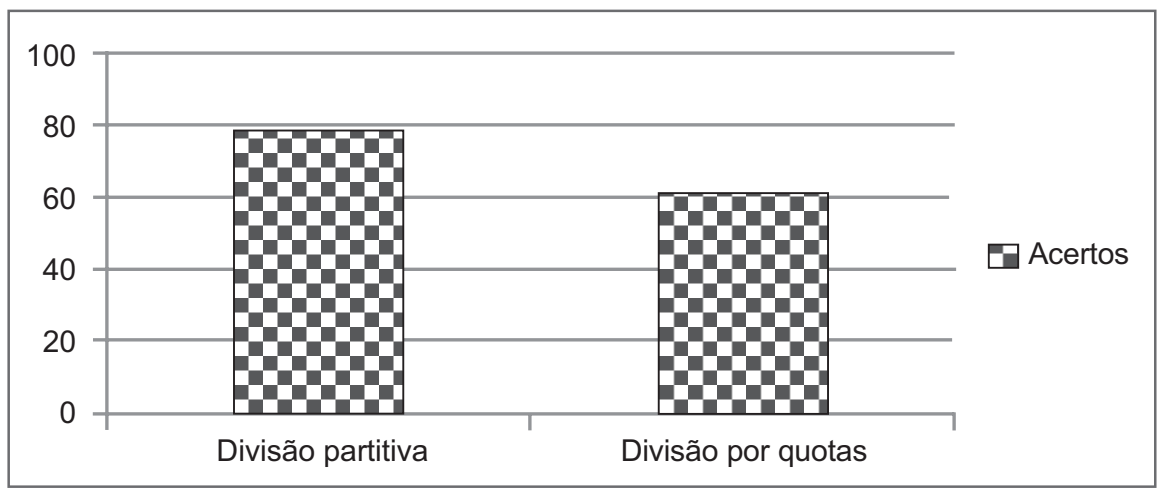


As taxas de acerto nas duas condições foram submetidas à análise, por meio do teste Wilcoxon. Verificou-se diferença estatisticamente relevante entre as duas condições experimentais $(\mathrm{Z}=2,15, \mathrm{p}=0,015)$.

No grupo controle (participantes adultos), o desempenho foi equivalente nas duas condições experimentais, não havendo nenhuma ocorrência de erro. Os resultados parecem indicar que, quando não há controle da complexidade gramatical dos enunciados, as crianças solucionam mais facilmente os enunciados de divisão partitiva do que os de divisão por quotas. Essa variável, no entanto, não interfere no desempenho dos adultos.

\section{Discussão}

A diferença de desempenho dos alunos nos dois tipos de problemas, com maior número de acertos nos problemas de divisão partitiva é um achado compatível com os resultados de Fischbein et al. (1985), Correa, Nunes \& Bryant (1998), Selva (1998), Kornilaki \& Nunes (1997,2005) eCorrea (2004), que também encontraram evidências de que os problemas partitivos são mais facilmente solucionados pelas crianças do que os de divisão por quotas. No entanto, dada a ampla diversidade de estruturas gramaticais utilizadas nos problemas deste experimento, entre as quais a presença de ambiguidade semântica referente à quantidade de objetos ser considerada (e.g. primeiro enunciado de divisão partitiva da tabela 1), não é possível precisar se a estrutural gramatical influenciou o desempenho dos alunos ou não. Dessa forma, decidimos conduzir um segundo experimento, no qual os dois tipos de problemas em questão foram linguisticamente estruturados da mesma maneira, evitando complexidades gramaticais que pudessem influenciar no desempenho dos alunos. 


\subsection{Experimento 2}

Havendo padronização da estrutura gramatical na elaboração de problemas de divisão partitiva e divisão por quotas, a diferença de desempenho dos alunos para as duas estruturas seria mantida? Para responder essa pergunta, elaboramos problemas de divisão, com uniformização da estrutura gramatical dos enunciados, mantendo a mesma complexidade gramatical nos dois tipos de divisão. Buscamos identificar nos dados usados no experimento 1 questões gramaticais que poderiam dificultar a interpretação dos enunciados. A partir dessa análise, controlamos, nos enunciados alvo usados no experimento 2, a complexidade da estrutura sintática (e suas possíveis interpretações semânticas) com objetivo de reduzir a complexidade gramatical e possíveis problemas de processamento. A variável dependente medida foi taxa de acerto.

Partimos, portanto, da hipótese de que a complexidade gramatical dos enunciados matemáticos de divisão afeta a resolução do problema, prevendo-se que a diferença entre as taxas de acertos nas duas condições (divisão partitiva e divisão por quotas) seriam menores no experimento 2 , em comparação com as taxas obtidas no experimento1.

Participantes

As mesmas crianças do $2^{\circ}$ ano do Ensino Fundamental que participaram do experimento 1 realizaram o experimento 2. O grupo controle, formado por adultos, também foi o mesmo do experimento anterior. Este experimento foi aplicado 10 dias após o experimento 1.

\section{Estímulos}

Foram utilizados 18 enunciados matemáticos, sendo 2 de treino, 8 distratores e 8 itens experimentais. Como no experimento 1, os itens distratores eram compostos por enunciados de adição, subtração e multiplicação. Nos itens experimentais, a variável independente 
manipulada foi o tipo de raciocínio matemático - 4 itens para divisão partitiva e 4 para divisão por quotas. Todos os participantes tiveram acesso à mesma lista de estímulos, mas em ordens de apresentação distintas.

Todos os itens experimentais seguiram a mesma organização estrutural, de acordo com a composição proposta por Gerofsky (1996), seguindo também o mesmo padrão informacional, como exemplificado abaixo: uma sentença introdutória de acomodação pragmática (1), uma primeira proposição formada por duas sentenças coordenadas, ambas no passado do modo indicativo (2), uma segunda proposição formada por duas sentenças subordinadas no presente do indicativo com o quantificador cada próximo ao final da sentença (3), e uma pergunta retórica de comando (4).

Quadro 2: organização estrutural dos estímulos usados no experimento 2.

\begin{tabular}{|l|l|l|}
\hline \multicolumn{1}{|c|}{ Sentenças } & \multicolumn{1}{c|}{ Divisão partitiva } & \multicolumn{1}{c|}{ Divisão por cota } \\
\hline 1 (set-up) & Antônia adora bala & Gustavo gosta de bola de gude \\
\hline $\begin{array}{l}\text { 2(componente } \\
\text { informativo) }\end{array}$ & $\begin{array}{l}\text { Ela comprou 14 balas e } \\
\text { precisa guardá-las em 2 } \\
\text { potes. }\end{array}$ & $\begin{array}{l}\text { Ele ganhou 1o bolinhas e quer } \\
\text { separá-las em saquinhos. }\end{array}$ \\
\hline $\begin{array}{l}\text { 3 (componente } \\
\text { informativo) }\end{array}$ & $\begin{array}{l}\text { Antônia não sabe qual } \\
\text { o número de balas ela } \\
\text { precisa colocar em cada } \\
\text { pote. }\end{array}$ & $\begin{array}{l}\text { Gustavo não sabe qual o número } \\
\text { de saquinhos ele precisa para } \\
\text { colocar 2 bolinhas em cada } \\
\text { saquinho. }\end{array}$ \\
\hline 4 (questão) & Você pode ajudá-la? & Você pode ajudá-lo? \\
\hline
\end{tabular}

Como no experimento 1, o teste foi aplicado em duas sessões, contendo 9 enunciados cada, apresentados em dias diferentes, com o intuito de reduzir o número de itens aos quais os alunos foram expostos e, consequentemente, reduzir os efeitos de cansaço e fadiga. Controlaram-se também as razões numéricas utilizadas nos itens experimentais. Apresentamos a seguir exemplos dos itens utilizados. 
Quadro 3: exemplo de estímulos usados no experimento 2.

\begin{tabular}{|c|l|}
\hline Tipo de divisão & \multicolumn{1}{c|}{ Enunciado } \\
\hline \multirow{5}{*}{ Divisão partitiva } & $\begin{array}{l}\text { Antônia adora bala. Ela comprou 14 balas e precisa guardá- } \\
\text { las em 2 potes. Antônia não sabe qual o número de balas ela } \\
\text { precisa colocar em cada pote. Você pode ajudá-la? }\end{array}$ \\
\cline { 2 - 2 } & $\begin{array}{l}\text { Giovana adora conchinha. Ela achou 10 conchinhas na } \\
\text { areia e quer usá-las para fazer 5 colares. Giovanna não sabe } \\
\text { qual o número de conchinhas ela precisa colocar em cada } \\
\text { colar. Você pode ajudá-la? }\end{array}$ \\
\hline \multirow{5}{*}{ Divisão por quotas } & $\begin{array}{l}\text { Henrique coleciona carta Pokémon. Ele possui 12 cartas e } \\
\text { precisa arrumá-las em envelopes. Henrique não sabe qual é } \\
\text { o número de envelopes ele precisa para colocar 4 cartas em } \\
\text { cada envelope. Você pode ajudá-lo? }\end{array}$ \\
\cline { 2 - 2 } & $\begin{array}{l}\text { Felipe adora adesivo. Ele comprou 6 adesivos e quer } \\
\text { colocá-las em cartolinas. Felipe não sabe qual o número } \\
\text { de cartolinas ele precisa para colar 3 adesivos em cada } \\
\text { cartolina? Você pode ajudá-lo? }\end{array}$ \\
\hline
\end{tabular}

\section{Procedimento}

Adotou-se o mesmo procedimento realizado no experimento 1. Os alunos foram instruídos a resolver os enunciados por meio de desenhos, e realizaram a atividade de forma autônoma, sem mediação do professor. Não foi estabelecido limite de tempo e os alunos levaram, em média, 45 minutos para resolver cada parte do teste. $\mathrm{O}$ grupo controle realizou as duas partes do teste no mesmo momento e levou cerca de 20 minutos para concluir a tarefa.

\section{Resultados}

Houve $81,25 \%$ de acerto nos itens de divisão partitiva e $78,75 \%$ nos que envolviam a divisão por quotas, o que indica alto percentual de acerto nas duas condições. A análise estatística dos dados foi feita através do teste Wilcoxon e não houve diferença significativa entre as condições experimentais $(\mathrm{Z}=0,68, \mathrm{p}=0,24)$. 
Gráfico 2: Taxa de erros e acertos nos dois tipos de enunciados

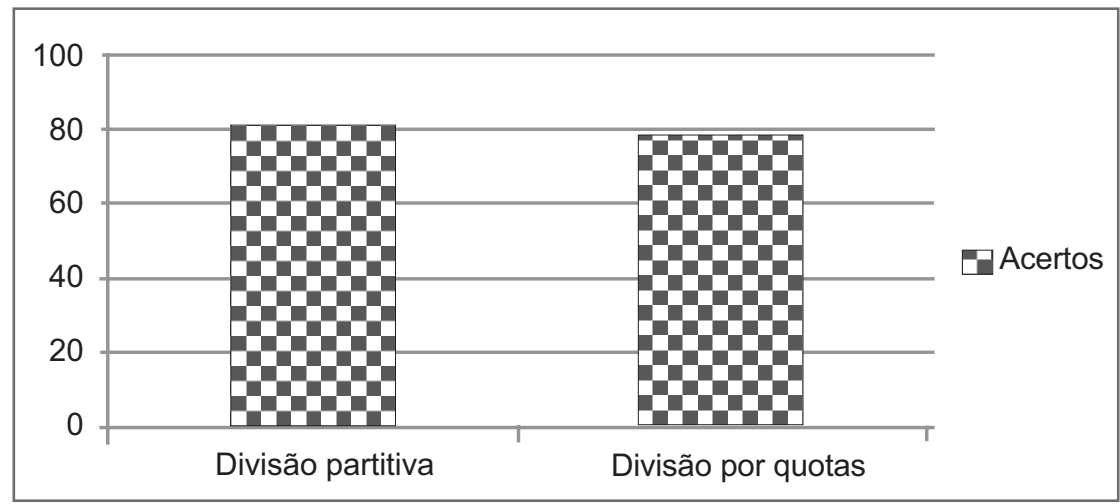

Portanto, verificamos que, quando a estrutura informacional e a complexidade gramatical dos enunciados é controlada, o desempenho dos alunos em tarefas de resolução de problemas de divisão partitiva e de divisão por quotas é equivalente. Desse modo, os resultados obtidos são compatíveis com os achados de Hill (1952), Brown (1981 $\left.{ }^{\mathrm{a}}\right)$, Burton (1992) e Downtown (2009), segundo os quais a acurácia do processo de resolução dos problemas de divisão independe do conceito de divisão atrelado aos enunciados. Por mais que tenha havido um pequeno percentual maior de acertos nos problemas partitivos, nossos resultados não permitem, assim como os de Correa (2004), corroborar as reflexões de Fischbein et al. (1985) de que a divisão partitiva seja o modelo intuitivo de divisão.

No que tange à influência da linguagem no desempenho matemático, nossos achados são compatíveis com os encontrados por Abedi \& Lord (2001) e Correia (2004), segundo os quais a complexidade gramatical dos enunciados influencia o processo de extração de informação dos problemas e sua, consequente, resolução. A diferença entre os resultados dos experimentos 1 e 2 corrobora a influência da complexidade da linguagem no desempenho matemático. 


\subsection{Comparação dos resultados}

Contrastou-se o número de acertos em cada uma das condições nos dois experimentos, por meio do teste Wilcoxon. Para a condição de divisão partitiva, não houve diferença significativa $(Z=1,28$; $\mathrm{p}=0,09)$. No entanto, a diferença foi significativa para a condição de divisão por quotas $(\mathrm{Z}=2,07 ; \mathrm{p}=0,016)$. Os resultados indicam que, nos problemas de divisão partitiva, cuja estruturação já segue um padrão organizacional prototípico e estruturas linguísticas mais simples, o controle do aspecto gramatical não altera (i.e. facilita ou dificulta) o desempenho dos alunos. No entanto, nos problemas de divisão por quotas, nos quais, geralmente, o enunciado não um segue um padrão estrutural e no qual são usadas diferentes estruturas linguísticas, dentre elas estruturas de alto custo, a uniformização gramatical contribui para que os alunos tenham melhor desempenho na resolução dos problemas.

O gráfico a seguir ilustra a média de respostas corretas por condição nos dois experimentos, ressaltando que só se obteve diferença estatisticamente significativa no contraste de desempenho na condição de divisão por quotas.

Gráfico 3: Média de respostas corretas nos dois experimentos

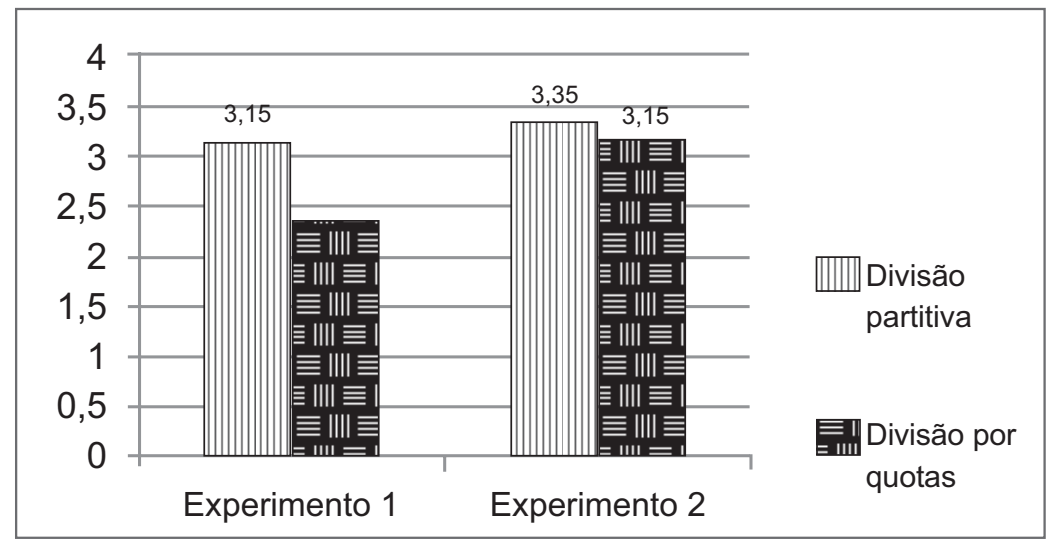


Assim como no experimento 1, o desempenho do grupo controle foi equivalente nas duas condições e não houve nenhuma ocorrência de erro de resolução.

Os resultados obtidos indicam que, no $2^{\mathrm{o}}$ ano do ensino fundamental, o papel da linguagem, no desempenho em tarefas de resolução de situações-problema que envolvem a operação da divisão, é bastante representativo. A comparação entre os resultados obtidos nos dois experimentos realizados sugere que o baixo desempenho das crianças em matemática pode criar uma "ilusão" de déficit matemático, quando, na verdade, o déficit pode ser de linguagem, com as crianças apresentando dificuldades no processamento e interpretação de enunciados gramaticalmente complexos. Essa dificuldade leva a uma integração "errônea" das informações necessárias para a resolução de problemas matemáticos com maior demanda cognitiva.

\section{Considerações finais}

Os resultados da pesquisa apresentada indicam que a dificuldade dos alunos em problemas de divisão pode ser reduzida com o controle da estrutura linguística dos enunciados, o que mostra o papel fundamental da observação de variáveis linguísticas na aferição de conhecimento matemático e na elaboração de materiais didáticos. Os resultados encontrados contribuem também para a investigação teórica acerca da interface linguagem-matemática, mais especificamente sobre o papel da gramática (língua-interna) em tarefas de resolução de tarefas de situações-problema. Do ponto de vista aplicado, a discussão dos resultados propõe reflexões sobre aspectos gramaticais que precisam ser considerados na elaboração de enunciados matemáticos e na avaliação das respostas dos alunos. 
Assumindo, assim como Kleiman (1997), que é também papel do professor criar oportunidades que permitam o desenvolvimento de processos cognitivos que levem à compreensão, a proposta de conduzir investigações experimentais, de caráter psicolinguístico, tem potenciais aplicações no campo do ensino e pode contribuir para o desenvolvimento de práticas pedagógicas nas quais o aluno seja capaz de conectar sua linguagem, seu conhecimento e suas experiências pessoais com a linguagem da classe e da área de conhecimento que se está trabalhando (CÂNDIDO, 2011).

\section{Referências}

ABEDI, J.; LORD, C.; HOFSTETTER, C. Impact of selected background variables on students' NAEP math performance. Los Angeles: UCLA Center for the Study of Evaluation/National Center for Research on Evaluation, Standards, and Student Testing, 1998.

ABEDI, J.; LORD, C. The Language Factor in Mathematics Tests. Applied Measurement In Education, 14 vol. 3, 219-234, 2001.

BARCELLOS, J. S.; RODRIGUES, E. dos S.; RODRIGUES, C. A. N. Esse é mais difícil por causa das palavras: uma investigação psicolinguística acerca do papel da linguagem na resolução de problemas matemáticos de divisão. 2017. Dissertação (Mestrado)-Pontifícia Universidade Católica do Rio de Janeiro, Departamento de Letras, 2017.

BRITO, M. R. F; FINI, L. D. T.; GARCIA, V. J. N. Um Estudo exploratório sobre as relações entre o raciocínio verbal e o raciocínio matemático - Pro-Posições, v. 5, n. 1, p. 37-44. 1994.

BROWN, M. Number Operations. In HART, K. M; MURRAY, J. Children's Understanding of Mathematics: 11-16 (Ed.), 1981a. 
BROWN, M. Levels of Understanding of Number Operations, Place-Value and Decimals in Secondary School Children - PH.d.Thesis. University of London, Chelsea College, 1981b.

BURTON, G. Young children's choices of manipulatives and strategies for solving whole number division problems. Focus on Learning Problems in Mathematics, v. 14, p. 2-57, 1992.

CÂNDIDO, P. T. Comunicação em Matemática. In: SMOLE, K. S.; DINIZ, M. I. (Org.). Ler, escrever e resolver problemas: habilidades básicas para aprender Matemática. Porto Alegre: Artmed. 2001, p. 15-28.

CARPENTER, T. et al. Children's Mathematics: Cognitively Guided Instruction. Portsmouth, NH: Heinemann/NCTM, 1999.

CORREIA, D. V. M. Complexidade sintáctica: Implicações na compreensão de enunciados de exercícios de Matemática. Actas do XX Encontro Nacional da Associação Portuguesa de Linguística, Lisboa, APL, p. 445-469, 2004.

. Estudos experimentais sobre leitura e compreensão de problemas verbais de matemática. Tese de doutorado. Universidade de Lisboa, Lisboa, 2013.

CORREA, J.; NUNES, T.; BRYANT, P. Young children's understanding of division: the relationship between division terms in a non-computational task. Journal of Educational Psychology, v. 90, p. 321-329, 1998.

DOWNTON, A. It seems to matter not whether it is partitive or quotitive division when solving one step division problems. In R. HUNTER, B. BICKNELL $\&$ T. BURGESS (Eds), Crossing divides Proceedings of the $32 n d$ annual conference of the Mathematics Education research Group of Australasia, v. 1, p. 161-168. Palmerston North, NZ: MERGA, 2009.

FISCHBEIN, E.; DERI, M.; MARINO, M. The role of implicit models in solving verbal problems in multiplication and division. Journal for Research in Mathematics Education, v. 16, p. 3-17, 1985.

FRUET, H. Todos podem compreender. Nova escola. São Paulo, agosto/2003, $\mathrm{n}^{\mathrm{o}} 164$, p. 38-39, 2003. 
GEROFSKY, S. A linguistic and narrative view of word problems in mathematics education. For the Learning of Mathematics, v. 16, n. 2, p. 36-45, 1996.

GIBSON, E. The dependency locality theory: a distance-based theory of linguistic complexity. In Y. MIYASHITA, A.; MARANTZ, \& W. O’Neil (Eds), Image, language, brain, 95-126. Cambridge, MA: MIT Press.

GUNDERSON, A. G. Thought-patterns of young children in learning multiplication and division. Elementary School Journal, v. 55, p. 453-461, 1955.

HILL, E. H. Study of Third, Fourth, Fifth and Sixth Grade Children's Preferences and Performances on Partition and Measurement Division Problems - State University of Iowa. Dissertation Abstracts vol. 12, 1952.

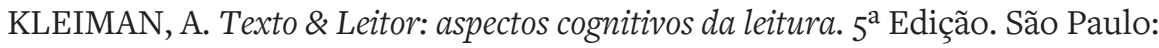
Pontes, 1997.

KORNILAKI, E. \& NUNES, T. Generalising Principles in spite of Procedural Differences: Children's Understanding of Division. Cognitive Development, v. 20, p. 388-406, 2005 .

KRUTETSKII, V. A. The psychology of mathematical abilities in scoolchildren. (TELLER,J. (Trad.), KILPATRICK, J.EWIRSZUP, I. (Eds)). Chicago:University of Chicago Press. 1976.

LORENSATTI, E. J. C. Linguagem matemática e língua portuguesa: diálogo necessário na resolução de problemas matemáticos. Revista Conjectura, v. 14, n. 2, p. 89-99, maio/ago. 2009.

MAMEDE, E.; VASCONCELOS, I. The Inverse Relation Between the Size and the Number of Parts. Journal of the European Teacher Education Network. vol. 11, p. 86-98, 2016.

MARTINELLO, M. Linguistic Complexity, Schematic Representations, and Differential Item Functioning for English Language Learners in Math Tests. Educational Assessment, 14:3-4, 160-179, 2009.

MAYER R. E. Thinking, Problem Solving, Cognition Second Edition. New York: Freeman, 1992. 
SELVA, A. C. V. Discutindo o uso de materiais concretos na resolução de problemas de divisão. In: SCHLIEMANN \& CARRAHER (Orgs.) A compreensão de conceitos aritméticos. Campinas, SP: Papirus, 1998.

WARREN, T. \& GIBSON, E. The influence of referential processing on sentence complexity. Cognition, 85, 79-112, 2002.

ZWENG, M. J. Division problems and the concept of rate. Arithmetic Teacher, v. 11, p. 547-556, 1964.

\section{Como citar}

BARCELLOS, Jéssica; RODRIGUES, Erica dos Santos; RODRIGUES, Cilene. O papel da língua na resolução de enunciados matemáticos. Revista da Abralin, v. 17, n. 1, p. 192-224, 2018.

Recebido 17/10/2018 e aceito em 12/01/2019. 